\title{
Biotransformation and metabolic profile of catalpol with human intestinal microflora by Ultra-performance liquid chromatography coupled with quadrupole time-of-flight mass spectrometry
}

Jin-hua Tao ${ }^{1}$, Min Zhao ${ }^{2}$, Dong-geng Wang ${ }^{1}$, Chi Yang ${ }^{1}$, Le-Yue Du² ${ }^{2}$, Wen-qian

$$
\text { Qiu }^{1} \text {, Shu Jiang }{ }^{2}
$$

1 School of Pharmacy, Nantong University, Nantong 226001, PR China

2 Jiangsu Collaborative Innovation Center of Chinese Medicinal Resources

Industrialization, Nanjing University of Chinese Medicine, 138 Xianlin Road,

Nanjing 210023, PR China

*Corresponding author.

Jin-hua Tao, Ph.D

Nantong University, 19 Qixiu Road, Nantong 226001, PR China

Tel. / fax: +8651385051716

E-mail address: taojinhua2000@163.com (J.H. Tao) 


\begin{abstract}
Traditional Chinese medicine (TCM) has been used in clinical practice for thousands of years. Catalpol, an iridoid glucoside, abundantly found in the root of the common used herb medicine Rehmannia glutinosa Libosch, has been reported to show various biological effects and pharmacological activities. After oral administration, the active ingredient might have interactions with the intestinal bacteria, which could help unravel how the medicine was processed in vivo. In this work, different pure bacteria from healthy human feces were isolated and used to $\begin{array}{llll}\text { bioconvert } & \text { catalpol. } & \text { Ultra } & \text { performance }\end{array}$ chromatography/quadrupole-time-of-flight mass spectrometry (UPLC-Q-TOF/MS) technique combined with Metabolynx ${ }^{\mathrm{TM}}$ software was applied to analyze catalpol metabolites. Compared with blank samples, parent compound (M0) and four metabolites (M1-M4) were detected and tentatively identified based on the characteristics of their protonated ions. The metabolites were likely to be: catalopl aglycone (M1), acetylated catalopl (M2), dimethylated and hydroxylated catalpol aglycone (M3), nitrogen-containing catalpol aglycone (M4). M1 and M4 were generated in the majority of the samples like Bacteroides sp. 45. M3 was obtained in several bacterial samples like Enterococcus sp. 8-2 and M2 was detected only in the sample of Enterococcus sp. 43-1. To our knowledge, the metabolic routes and metabolites of catalopl produced by human intestinal bacteria were all firstly reported.
\end{abstract}


Keywords: Catalopl, Human intestinal bacteria, UPLC-Q-TOF/MS, Metabolites

\section{Introduction}

Rehmannia (known as Dihuang) has long been used extensively as a traditional health food in East Asia and displays numerous biological activity. Catalpol, an iridoid glucoside contained richly in the roots of the small flowering plant species Rehmannia glutinosa Libosch, has been shown to have antioxidation, anti-inflammation, anti-apoptosis, anti-brain ischemia, anti-senile dementia, and other neuroprotective properties and plays a role in neuroprotection against hypoxic/ischemic injury, Alzheimer disease and Parkinson's disease both in vivo and in vitro models. [1-3]. It has protective effects against $\mathrm{H}_{2} \mathrm{O}_{2}$-induced oxidative damage in astrocytes [4] and may possess therapeutic potential against LPS-induced acute systemic inflammation by attenuating NF-KB activation and protecting mitochondrial function in cerebral cortex and hippocampus [2,5]. Catalpol has been reported to rescue neurons from kinds of damage in vitro and in vivo. It improved memory and protected the forebrain neurons from neurodegeneration through increasing brain derived neurotrophic factor (neuronotrophic) (BDNF) expression [6], protected primary cultured cortical neurons induced by Abeta(1-42) through a mitochondrial-dependent caspase pathway [7]. Studies also have shown that catalpol was a potential agent for the prevention of neurons apoptosis by regulating NO and iNOS increase in ERK-mediated neurodegenerative disorders [8].

The microbial community in the gut contains three domains of life: bacteria, archaea and eukarya (fungi, yeasts and protozoa), with the largest community residing 
in the colon. Indeed, the human colon harbors a highly complex microbial ecosystem of about $200 \mathrm{~g}$ living cells, at concentrations of $10^{12}$ microorganisms per gram gut content, the highest recorded for any microbial habitat. Together, all 6.5 billion humans on earth represent a gut reservoir of $10^{23}-10^{24}$ microbial cells, which is just five orders of magnitude less than the world's oceans $\left(10^{29}\right.$ cells) [9-10]. Therefore, the human gut constitutes a substantial habitat in our biosphere. The endogenous gastrointestinal microbial flora plays a fundamentally important role in human health and disease, yet this ecosystem remains incompletely characterized and its diversity poorly defined. The intestinal microbiota can therefore be regarded as a separate organ within the human host, which is capable of even more conversions than the human liver.

Most herbal medicines are administered orally and their components are inevitably brought into contact with intestinal microflora in the alimentary tract. Most components are metabolized by the intestinal bacteria before absorption from the gastrointestinal tract [11]. Intensive metabolism results in often low circulating levels of the original products, with the consequence that final health effects of botanicals are often related to specific active metabolites which are produced in the body rather than being related to the product's original composition. Understanding how such metabolic processes contribute to the in situ exposure is therefore crucial for the proper interpretation of biological responses [12].

However, little is known about the roles of human intestinal bacteria in the metabolic processes and biological activities of catalopl now. The aim of this work 
was to isolate different pure bacteria from human feces and investigate the capability of human gut bacteria to biotransform catalopl. To further clarify the metabolic profile of catalopl by the isolated human intestinal bacteria, a rapid and sensitive ultra performance liquid chromatography/quadrupole time-of-flight mass spectrometry (UPLC-Q-TOF/MS) with automated data analysis (MetaboLynx) was established to identify its metabolites [13]. The results probably provided helpful information for further in vivo metabolism and action mechanism research on catalopl.

\section{Experimental}

\subsection{Materials}

Catalopl standard substance (purity, >98\%) was purchased from Shanghai Winherb Medical S \&T Development Co. Ltd (Shanghai, China). The formic acid was obtained from Merck KGaA (Darmstadt, Germany). The HPLC-grade acetonitrile was purchased from TEDIA Company Inc. (Fairfield, USA). The distilled water was purified by an EPED super purification system (Nanjing, China). Other reagents were of analytical grade. AnaeroPack Rectangular Jars were purchased from Mitsubishi Gas Chemical Company INC (Japan). General anaerobic medium (GAM) was purchased from Shanghai Kayon Biological Technology Co. Ltd. (Shanghai, China).

\subsection{Organism}

The human fecal specimens were prepared according to previous methods [14]. 
Briefly, $4 \mathrm{~g}$ of human fresh feces from a healthy Chinese man (28-year-old, no smoking, no current medication, no use of antibiotics, and fasting before sampling) were collected in a plastic cup. The sample was then suspended in $20 \mathrm{ml}$ sterile physiological saline and homogenized adequately by a vortex-mixer. The fecal mixture was centrifuged for $10 \mathrm{~min}$ and the supernatant was then filtered with gauze. The suspension was used as a metabolic intestinal microflora fraction.

The bacterial mixture was diluted serially in aquae sterilisata and each of the dilutions was spread on the GAM agar plates which were incubated under anaerobic condition in anaerobic jars for $48 \mathrm{~h}$ at $37^{\circ} \mathrm{C}$. At last, about one hundred different types of bacterial colonies developed on plates were observed [15]. Each bacterium picked up from GAM agar plate was inoculated into $1.0 \mathrm{~mL}$ GAM broth and anaerobically incubated at $37{ }^{\circ} \mathrm{C}$ for $36 \mathrm{~h}$.

\subsection{Preparation of catalopl sample for analysis}

The standard solution was prepared by dissolving accurately weighed catalopl in methanol to give a final concentration of $3.0 \mathrm{mg} / \mathrm{mL}$. $0.1 \mathrm{mM}$ catalopl was inoculated into $0.9 \mathrm{~mL}$ of GAM broth with $0.1 \mathrm{~mL}$ precultured bacteria and anaerobically incubated at $37{ }^{\circ} \mathrm{C}$ for $36 \mathrm{~h}$. The sample was extracted twice with acetone, and the acetone layer dried under vacuum and dissolved with $0.3 \mathrm{~mL}$ methanol. The water layer was extracted twice with butanol, and the butanol layer dried under vacuum and dissolved with $0.3 \mathrm{~mL}$ methanol, combined with the acetone layer. The mixture was centrifuged at $12,000 \times \mathrm{g}$ for $10 \mathrm{~min}$ before being injected for. UPLC/MS analysis. 


\section{$2.4 U P L C-M S$ spectrometry}

Catalopl and its metabolites were separated and identified on a Waters ACQUITY UPLC system (Waters Corp., Milford, MA, USA) fitted with a Syncronis C 18 column $(100 \mathrm{~mm} \times 2.1 \mathrm{~mm}$ i.d., $1.7 \mu \mathrm{m}$; Thermo, USA). The chromatographic conditions were optimized to give good separation of the target compounds and avoid the interferences. The column temperature was maintained at $35{ }^{\circ} \mathrm{C}$. A gradient elution system was used with mobile phase A ( $0.1 \%$ formic acid in distilled water) and mobile phase $\mathrm{B}$ (acetonitrile) at a total flow rate of $0.4 \mathrm{ml} / \mathrm{min}$. The gradient program was started at $95 \%$ mobile phase A and $5 \%$ mobile phase $\mathrm{B}$, changed linearly to $10 \%$ mobile phase A and $90 \%$ mobile phase B for 11 min, and was held for $1 \mathrm{~min}$. The gradient was recycled back to $5 \%$ in $1 \mathrm{~min}$ for the next run. The injection volume was $5 \mu \mathrm{L}$. The retention time of catalopl was $0.91 \mathrm{~min}$.

For analysis, a Waters ACQUITY Synapt mass spectrometer (Waters Corp., Manchester, UK) connected to the UPLC system via an electrospray ionization (ESI) interface was used. ESI in both positive and negative ion modes were attempted in the method development. Since the metabolites were more abundant in positive ion mode. The characterization of the metabolites was based on their ion fragmentation in $\mathrm{ESI}^{+}$ mode. In the full scan mass spectra, the protonated molecular ions $[\mathrm{M}+\mathrm{H}]^{+}$were more stable and exhibited higher abundance than $[\mathrm{M}+\mathrm{Na}]^{+}$, so the $[\mathrm{M}+\mathrm{H}]^{+}$was chosen as the precursor ion for MS/MS fragmentation analysis.

Product ion mass spectra were obtained at different desolvation temperature, 
source temperature, desolvation gas flow and capillary voltage to select maximum sensitivity for most constituents. The temperature of the ion source was maintained at $120{ }^{\circ} \mathrm{C}$ and desolvation temperature was $350{ }^{\circ} \mathrm{C}$. The gas $\left(\mathrm{N}_{2}\right)$ flows of cone and desolvation were 50 and $600 \mathrm{~L} / \mathrm{h}$, respectively. The capillary voltage used for the analysis of catalopl and its metabolites was $3.0 \mathrm{kV}$. All data collected in centroid mode were acquired using Masslynx ${ }^{\mathrm{NT}} 4.1$ software (Waters Corp., Milford, MA USA).

\section{Results and discussion}

\subsection{Metabolic profile of catalopl by the different human intestinal microflora}

A full overview of the catalopl metabolic profile was obtained after optimizing the UPLC and MS conditions. Using the positive ion electrospray tandem mass spectrometry and the Metabolynx ${ }^{\mathrm{TM}}$ program, catalopl (M0) and its four metabolites (Table 1) were detected in different bacteria samples compared with blank samples. These metabolites were likely to be: catalopl aglycone (M1), acetylated catalopl (M2), dimethylated and hydroxylated catalpol aglycone (M3) and nitrogen-containing catalpol aglycone (M4). M1 and M4 were obtained in the majority of samples of the human intestinal bacteria isolated from the feces in this study. However, M3 was generated in several bacterial samples like Enterococcus sp. 8-2 and M2 was detected only in the sample of Enterococcus sp. 43-1. The extracted ion chromatograms (XICs) from the total UPLC/MS chromatograms were shown in Fig. 1. 


\subsection{Identification of catalopl metabolites}

\subsubsection{Parent compound}

Catalopl (M0) was identified by comparing the UPLC retention time (0.91 $\mathrm{min})$, accurate MS at m/z $363.1292[\mathrm{M}+\mathrm{H}]^{+}$and MS/MS spectra with the authentic standard (Fig. 2a). As illustrated in the fragmentation pathway (Fig. 3a), the precursor ion $[\mathrm{M}+\mathrm{H}]^{+} \mathrm{m} / \mathrm{z} 363.1292$ afforded the catalopl aglycone product ion at m/z 201.3302. The product ions at $\mathrm{m} / \mathrm{z}$ 183.1218, 165.1009 and 147.2001 were formed from $\mathrm{m} / \mathrm{z}$ 201.3302 by loss of $\mathrm{H}_{2} \mathrm{O}, 2 \mathrm{H}_{2} \mathrm{O}$ and $3 \mathrm{H}_{2} \mathrm{O}$, respectively. The other characteristic fragments were m/z 137.0019 and 109.0021 corresponding to the loss of $\mathrm{CO}$ and $2 \mathrm{CO}$ from $\mathrm{m} / \mathrm{z} 165.1009$.

\subsubsection{Metabolite M1}

The retention time and fragment ion of metabolite M1 were at $2.95 \mathrm{~min}$ and $\mathrm{m} / \mathrm{z}$ 201.3302, respectively. Fragment ion at m/z 201.3302 was generated from M0 m/z 363.1292 by loss of the glucuronide group. According to the MS/MS spectrum and the fragmentation regularity (Fig. $2 b$ and Fig. $3 b$ ), the other fragment ions of M1 were also yielded in the mass spectrum of M0. Thus, M1 was identified as the aglycone of catalopl.

\subsubsection{Metabolite M2}

Metabolite M2 showed an UPLC profile with a retention time at $6.41 \mathrm{~min}$ (Fig. 2c). The fragment ion of M2 was at $\mathrm{m} / \mathrm{z} 243.4210$ which was 42 Da higher than M1 
and it was also fragmented to give $\mathrm{m} / \mathrm{z}$ 201.3302, 183.1218, 165.1009, 147.2001, 137.0019, 109.0021 (Fig. 3c). Therefore, M2 was identified as acetylated catalopl aglycone.

\subsubsection{Metabolite M3}

The fragment ion of M3 (retention time $4.35 \mathrm{~min}$ ) was at $\mathrm{m} / \mathrm{z} 247.4360$, which was 46 Da higher than M1 (Fig. 2d). Fragment ion at m/z 229.1017 was obtained from $\mathrm{M} 3$ by loss of $\mathrm{H}_{2} \mathrm{O}$. Then, the $\mathrm{m} / \mathrm{z} 229.1017$ lost $\mathrm{CH}_{2} \mathrm{O}$ and $2 \mathrm{CH}_{2} \mathrm{O}$ to give $\mathrm{m} / \mathrm{z}$ 199.0261 and 169.3016, respectively (Fig. 3d). So, M3 was identified as dimethylated and hydroxylated catalpol aglycone.

\subsubsection{Metabolite M4}

Metabolite M4 was detected as deprotonated molecular ion $[\mathrm{M}+\mathrm{H}]^{+}$at $\mathrm{m} / \mathrm{z}$ 200.2514 with retention time of 3.42 min (Fig. 2e), which was 1 Da lower than that of M1. The product ions at $\mathrm{m} / \mathrm{z} 182.3021$ and 164.0381 were formed by the elimination of $\mathrm{H}_{2} \mathrm{O}$ and $2 \mathrm{H}_{2} \mathrm{O}$ from $\mathrm{m} / \mathrm{z}$ 200.0514, respectively (Fig. 3e). It was reported that morroniside, geniposide and gardenoside were transformed to new nitrogen-containing compounds, mor-1 and mor-2, genipinine and gardenine, respectively, along with the known aglycones [16]. In common with these compounds, catalpol is also an iridoid glycoside. Based on the above analysis, the similar metabolic pathway was possible for catalpol and M4 was proposed to be a nitrogen-containing compound. 


\subsection{Proposed metabolic pathways of catalopl by different human intestinal bacteria}

According to our experimental results, the metabolic pathways of catalopl by human intestinal bacteria were proposed as shown in Fig. 4. After incubation with the isolated bacteria, M1 in the majority sample was identified as catalopl deglycosylated metabolite. Some other bacteria like Enterococcus sp. 43-1 showed moderate activities for the aglycone. From these results, we could deduce that catalopl was firstly metabolized to be catalopl aglycone by $\beta$-D-glucosidase from these bacteria, thereafter catalopl aglycone was metabolized to be acetylated and dimethylated and hydroxylated catalopl. It was reported that morroniside, a bioactive iridoid glycoside, could be firstly hydrolyzed to its aglycone. After the cleavage of B-ring of the aglycone, two steps of nucleophilic addition reaction took place to produce an $\mathrm{N}$-heterocycle derivative, and this derivative further dehydrated to form mor-1; and the product from the cleavage of A- and B-ring of the aglycone would produce mor-2 via a step of benzoin condensation and two steps of nucleophilic addition reaction [17]. Due to the similar chemical structure with morroniside, the metabolic pathway was possible for catalopl. Thus, we proposed nitrogen-containing catalopl aglycone was produced after the aglycone being released.

There are 100 trillion microbes in the human gastrointestinal tract with numbers increasing distally. These microbiota secrete a diverse array of enzymes (primarily for carbohydrate and protein fermentation) giving them substantial metabolic potential which can have major implications for drug stability [18]. The set of the colonic 
microbial community genes that code for metabolic enzymes is highly diverse and redundant to ensure survival under fluctuating nutritional conditions [12]. The hydrolytic function is essential for providing bacteria with carbon and energy from the released sugars and/or oligosaccharides. The activities of the various hydrolases are mainly associated with the bacterial fraction in fecal samples [19,20]. While, methylation can greatly enhance the medicine metabolic stability [21] and improve its lipophilicity than the prototype drug.

The naturally occurring iridoid catalpol is a Taq DNA polymerase inhibitor. However, its poor lipophilicity might account for the lack of biological activity against human solid tumor cell lines. The traditional prodrug approach by means of peracetylation of the free hydroxyl groups led to a compound, which showed a marginal growth inhibition against the most sensitive cell line A2780 (ovarian cancer) [22]. In this study, acetylated product of catalpol also could be produced by human intestinal bacteria. Compared with the traditional prodrug approach, biotransformation way might have its special advantages.

\section{Conclusion}

In this paper, a method using ultraperformance liquid chromatography/quadrupole time-of-flight mass spetrometry (UPLC-Q-TOF/MS) with automated data analysis (MetaboLynx ${ }^{\mathrm{TM}}$ ) was established for search and identification of catalpol metabolites produced by human intestinal bacteria in vitro. Four catalpol metabolites were tentatively identified and the metabolic pathways were 
firstly proposed, respectively. To our knowledge, catalpol, a poor lipophilicity and the water-soluble component, has a low bioavailability. But biotransformation might improve the bioavailability [15]. Investigation of the metabolism of catalpol by intestinal bacteria in vitro could help understand the metabolism of the component after oral administration and would be useful for tracing the therapeutic effects of medications for certain disease. However, further studies were still needed to identify the enzyme system which contributed to the metabolism and reaction mechanisms by the intestinal bacteria.

\section{Acknowledgements}

This work was financially supported by the Nature Science Foundation of China (NO.81072996, 81102743) and the Priority Academic Programs Development of Jiangsu Higher Education Institutions (PAPD).

\section{References}

[1] H. Zhu, D. Wan, F. Zhang. Progress in studies of pharmacological action and mechanisms of catalpol on brain diease. China J. Chin. Mater. Med. 34 (2009): 2983-2986.

[2] Bi. Jing, B. Jiang, Z. Andrew, R.G. Zhao, L. Peng, J.A. Li. Catalpol inhibits LPS plus IFN-c-induced inflammatory response in astrocytes primary cultures. Toxicol. In Vitro. 27 (2013): 543-550. 
[3]B. Jiang, R.F. Shen, J. Bi, X.S. Tian, T. Hinchliffe, Y. Xia. Catalpol: a potential therapeutic for neurodegenerative diseases, Curr. Med. Chem. 22(2015): $1278-1291$.

[4] Z. Zhang, Y. Liu, B. Xue, L. Wei. Protective effects of catalpol against $\mathrm{H}_{2} \mathrm{O}_{2}$-induced oxidative damage in astrocytes. China J. Chin. Mater. Med. 34 (2009): 1955-1958.

[5] A. Zhang, S. Hao, J. Bi, Y. Bao, X. Zhang, L. An, B. Jiang. Effects of catalpol on mitochondrial function and working memory in mice after lipopolysaccharide-induced acute systemic inflammation. Exp. Toxicol. Pathol. 61(2009): 461-469.

[6] Z. Wang, Q. Liu, R. Zhang, S. Liu, Z. Xia, Y. Hu. Catalpol ameliorates beta amyloid-induced degeneration of cholinergic neurons by elevating brain-derived neurotrophic factors. Neuroscience, 163(2009): 1363-1372.

[7] J.H. Liang, J. Du, L.D. Xu, T. Jiang, S. Hao, J. Bi, B. Jiang. Catalpol protects primary cultured cortical neurons induced by Abeta(1-42) through a mitochondrial-dependent caspase pathway. Neurochem. Int. 55 (2009): 741-746.

[8] J. Bi, B. Jiang, S. Hao, A. Zhang, Y. Dong, T. Jiang, L. An. Catalpol attenuates nitric oxide increase via ERK signaling pathways induced by rotenone in mesencephalic neurons. Neurochem. Int. 54(2009): 264-270.

[9] B. Dridi , D. Raout, M. Drancourt. Archaea as emerging organisms in complex human microbiomes. Anaerobe. 17(2011):56-63.

[10] L. Vitetta, S. Hall, A.W. Linnane. Live probiotic cultures and the gastrointestinal 
tract: symbiotic preservation of tolerance whilst attenuating pathogenicity. Front Cell Infect Mi. 15 (2014):143-153.

[11] C. Alonso, M. Vicario, M. Pigrau, B. Lobo, J. Santos. Intestinal barrier function and the brain-gut axis. Adv. Exp. Med. Biol. 817(2014): 73-113.

[12] S. Possemiers, S. Bolca, W. Verstraete, A. Heyerick. The intestinal microbiome: A separate organ inside the body with the metabolic potential to influence the bioactivity of botanicals. Fitoterapia. 82 (2010): 53-56.

[13] J.M. Guo, C.F. Xue, E.X. Shang, J.A. Duan, Y.P. Tang, D.W. Qian.. Identification of hyperoside metabolites in rat using ultra performance liquid chromatography/quadrupole-time-of-flight mass spectrometry. J. Chromatogr. B. 879 ( 2011): 1987-1992.

[14] J.Y. Wan, P. Liu, H.Y. Wang, L.W. Qia, C.Z. Wang, P. Li, C.S.Yuan. Biotransformation and metabolic profile of American ginsengs aponins with human intestinal microflora by liquid chromatographyquadrupole time-of-flight mass spectrometry. J. Chromatogr. A. 1286 (2013): 83-92.

[15] H.G. Hur, J.O. Lay, R.D. Beger, J.P. Freeman, F. Rafii. Isolation of human intestinal bacteria metabolizing the natural isoflavone glycoside daidzin and genistin. Arch. Microbiol. 174(2000): 422-428.

[16] T. Sousa, R. Paterson, V. Moore, A. Carlsson, B. Abrahamsson, A.W. Basit. The gastrointestinal microbiota as a site for the biotransformation of drugs. Int. J. Pharm. 363 (2008): 10-25.

[17] X.N. Li, C.H. Huo, Q. Wang, X.W. Zhang, X.N. Sheng, L.T. Zhang. 
Identification of new metabolites of morroniside produced by rat intestinal bacteria and HPLC-PDA analysis of metabolites in vivo. J. Pharmaceut. Biomed. 45 (2007): 268-274.

[18] S. Unger, A. Stintzi, P. Shah, D. Mack, D.L. O'Connor .Gut microbiota of the very low birth weight infant. Pediatr. Res. 77(2015): 205-213.

[19] E. Altindis, T. Dong, C. Catalano, J. Mekalanos. Secretome analysis of vibrio cholerae type VI secretion system reveals a new effector-immunity pair. MBio. 6(2015): 2150-7511.

[20] Y. Yang, J.L. Millan, J. Mecsas, K. Guillemin. Intestinal alkaline phosphatase deficiency leads to lipopolysaccharide desensitization and faster weight gain. Infect Immun. 83( 2015): 247-258.

[21] X. Wen, T. Walle. Methylated flavonoids have greatly improved intestinal absorption and metabolic stability. Drug Metab. Dispos. 34( 2006): 1786-1792.

[22] C.R. Pungitore, L.G. Leon, C. Garcia, V.S. Martin, C.E. Tonn, J.M. Padron. Novel antiproliferative analogs of the Taq DNA polymerase inhibitor catalpol. Bioorg. Med. Chem. Lett. 17(2007): 1332-1335.

\section{Figure captions}

Fig. 1. UPLC/MS chromatograms of catalpol and its metabolites. (a) GAM with standard solution of catalpol sample, (b) Bacteroides sp. 45 sample, (c) Enterococcus sp. 8-2 sample, (d) Enterococcus sp. 43-1 sample. 
Fig. 2. UPLC-MS/MS spectra. (a) M0 (m/z 363), (b) M1 (m/z 201), (c) M2 (m/z 243), (d) M3 (m/z 247), (e) M4 (m/z 200).

Fig. 3. Proposed fragmentation pathways of M0-M4.

Fig. 4. Proposed metabolic pathways of catalpol by human intestinal bacteria. 
(a)

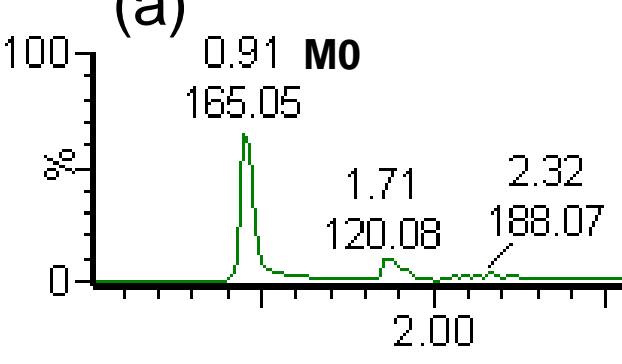

(b)$$
100
$$

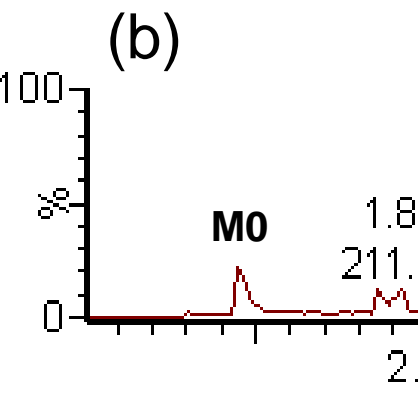

(c)

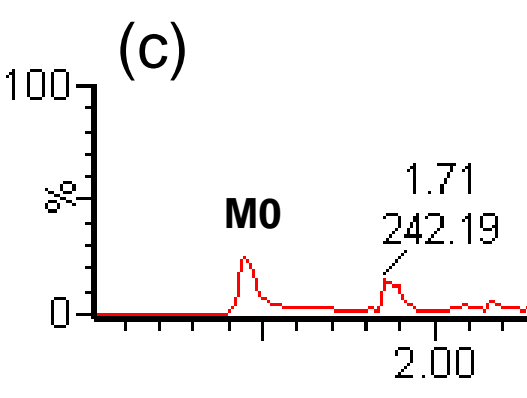

(d)

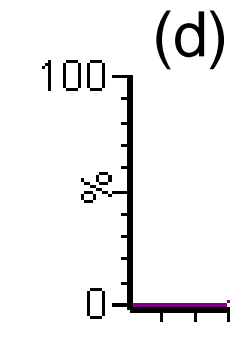

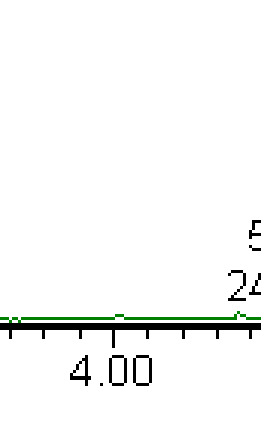

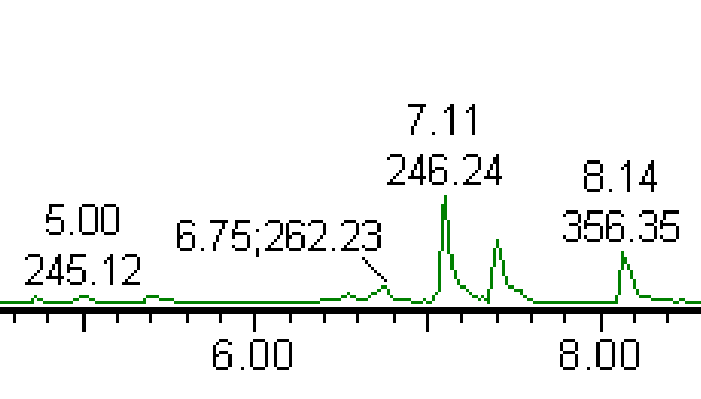

\subsection{1 282.28} 10.06
282.28 282.28 $363+201+243+247+200$ 20 282.28
10.00

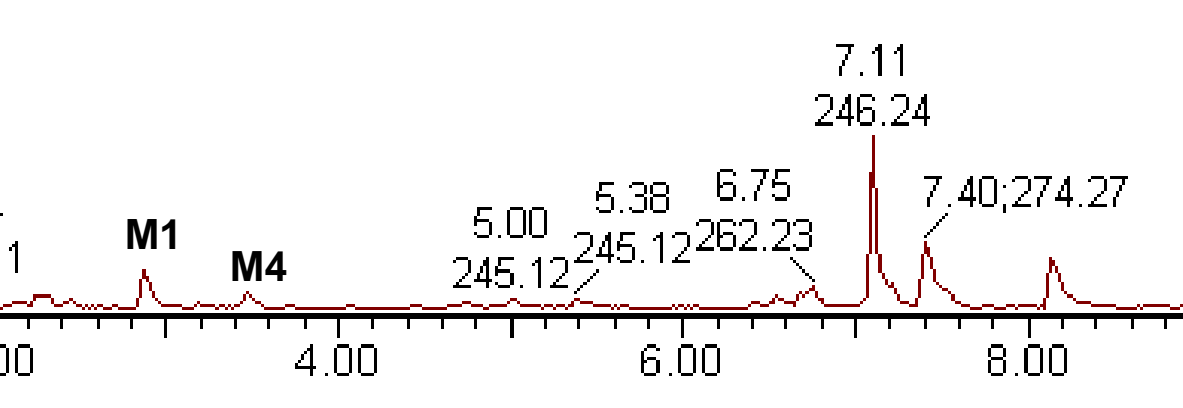

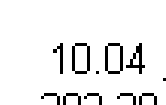

10.04
282.28

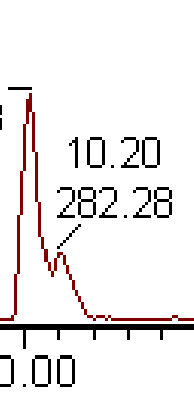

12.00
$1:$ TOF MS ES +
$363+201+243+247+200$
$2.87 \mathrm{e} 3$ 


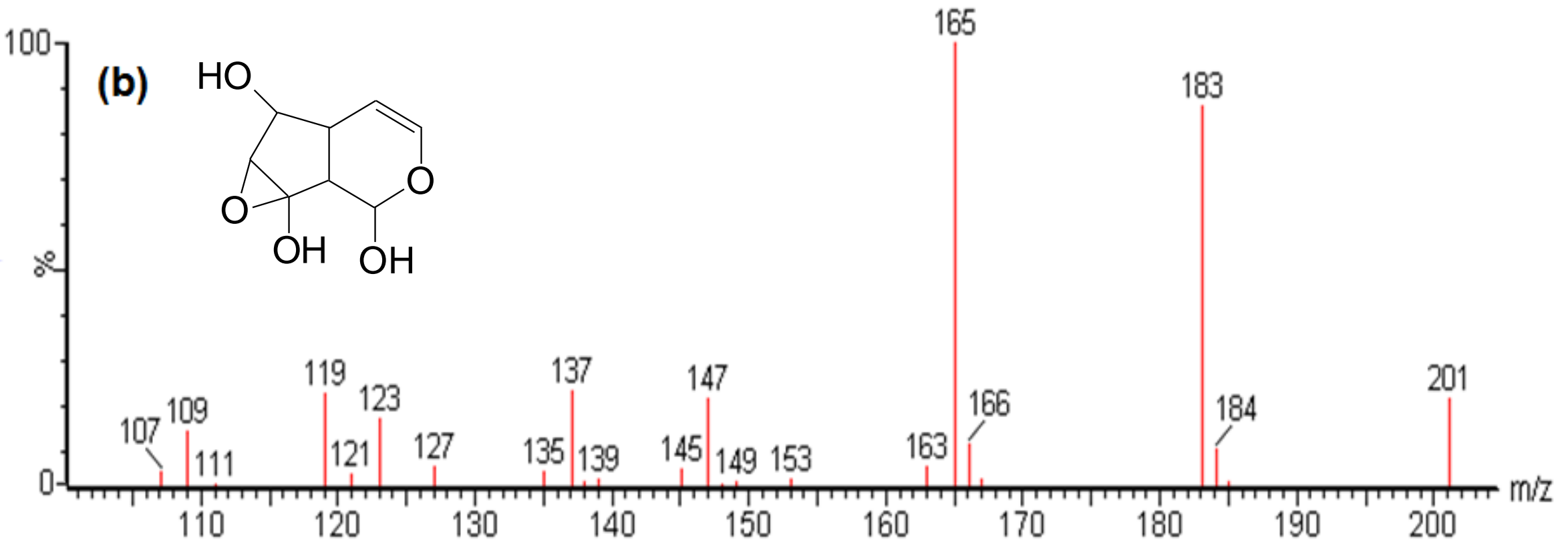


(a)

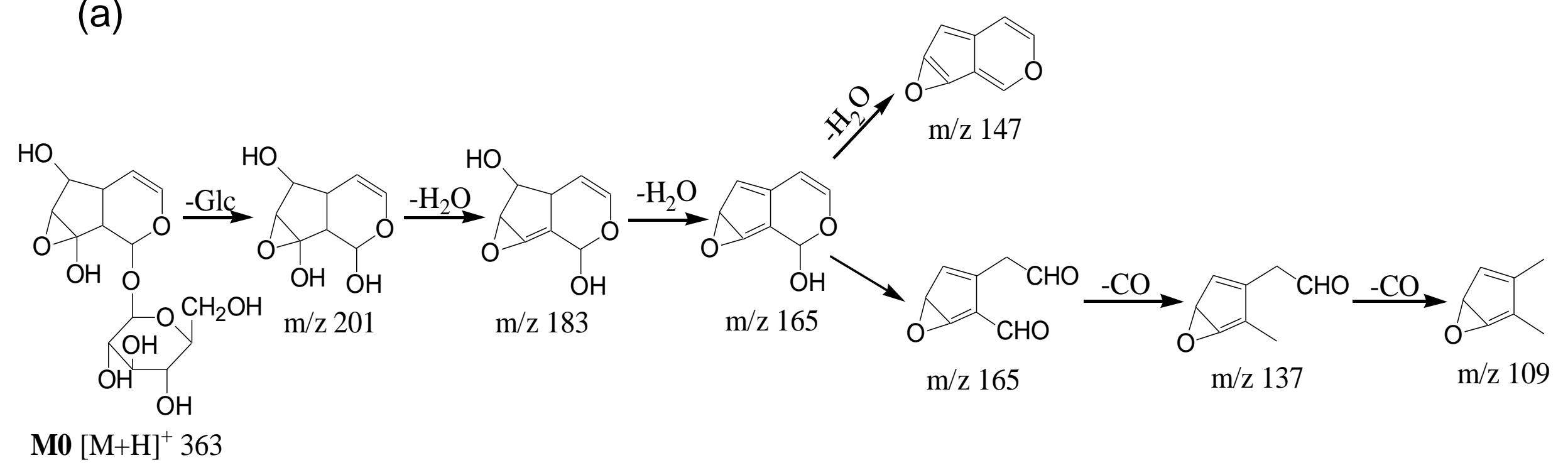


(b)

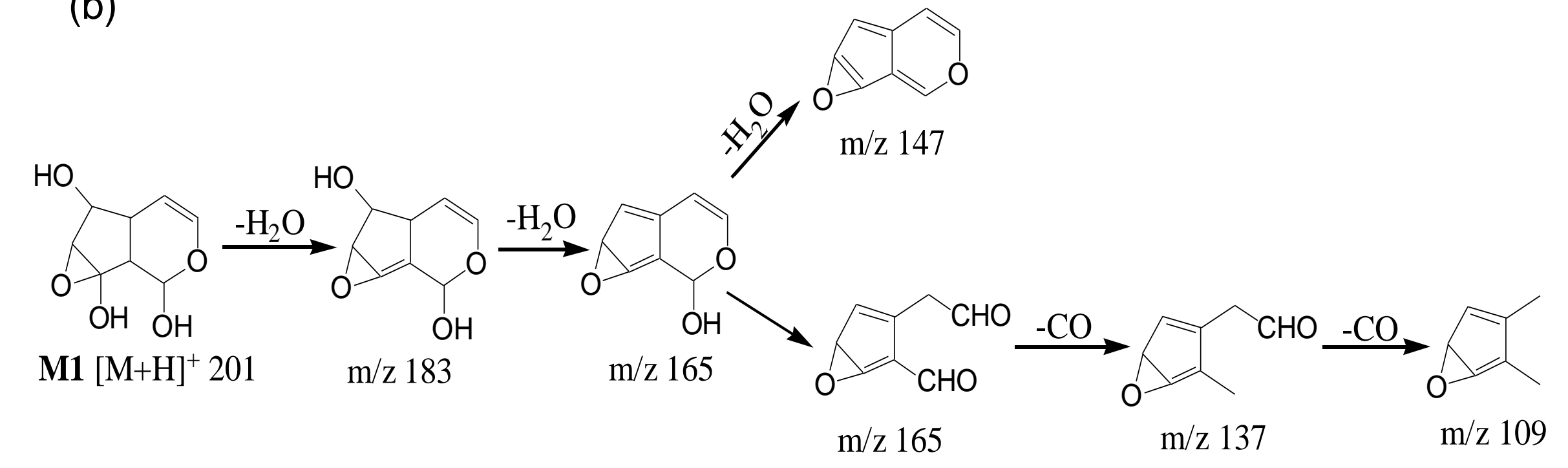


(c)

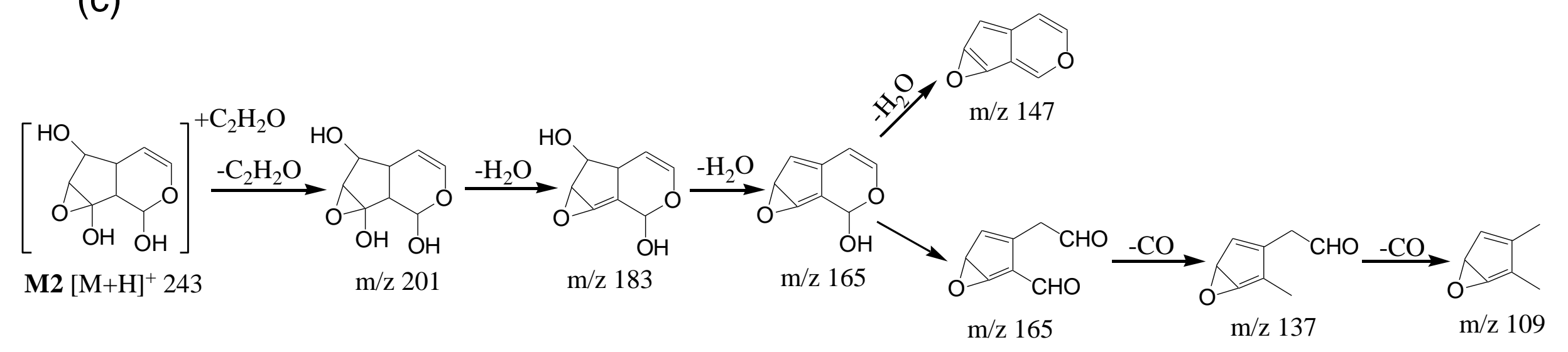


(d)

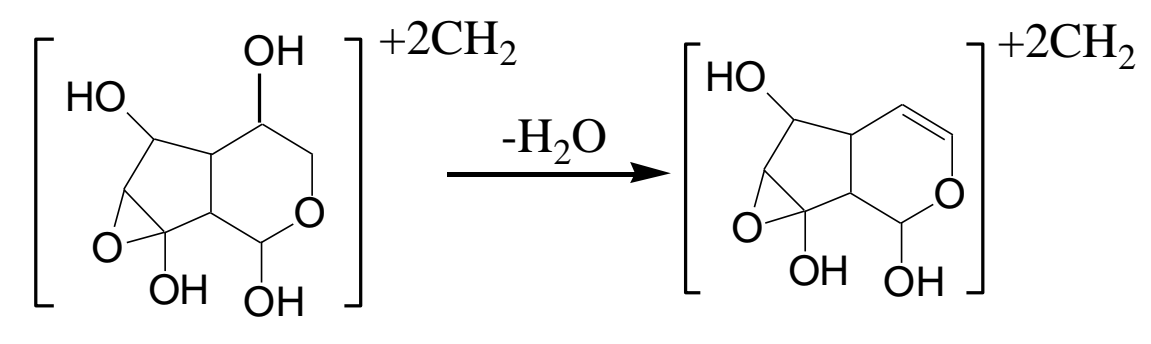

M3 $[\mathrm{M}+\mathrm{H}]^{+} 247 \quad$ m/z 229 
(e)

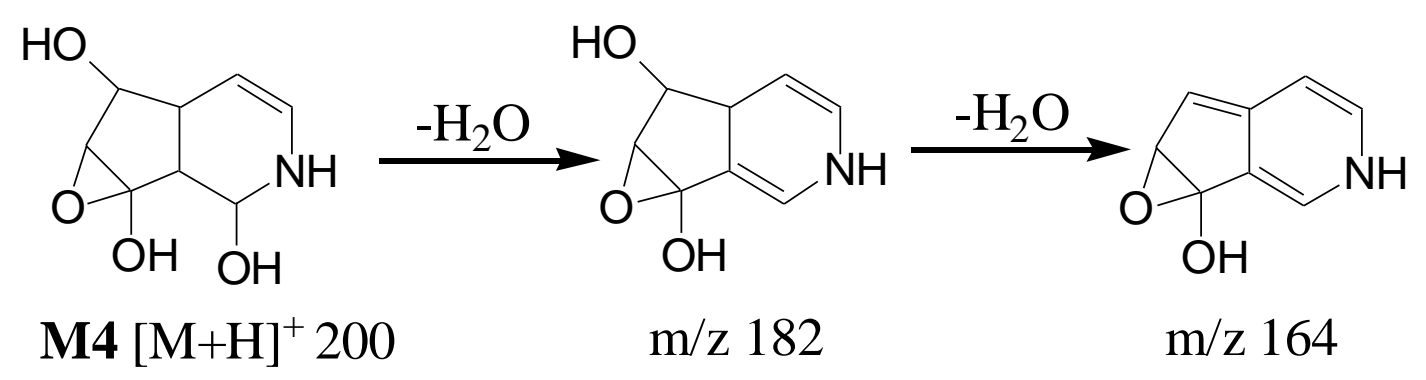

Fig. 3 

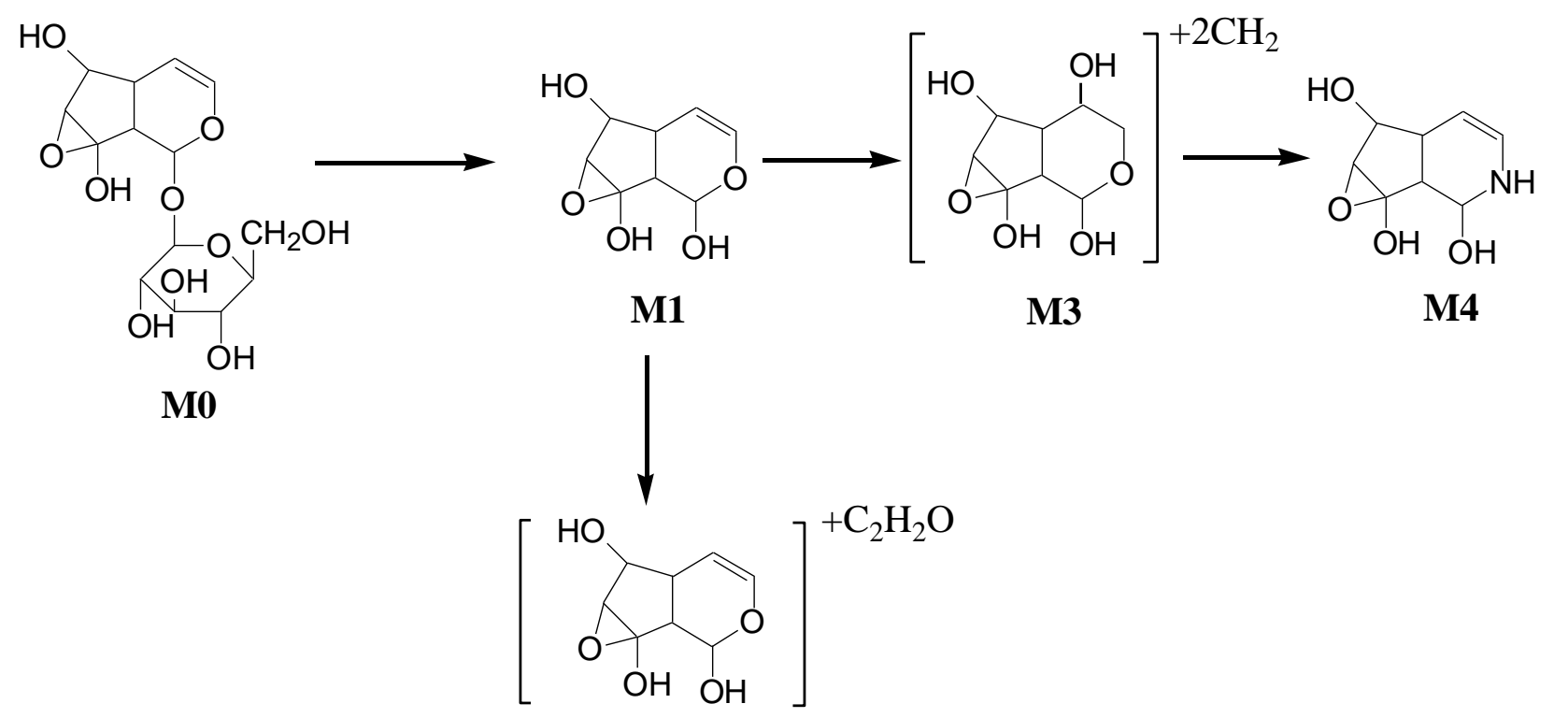

M2

Fig. 4 


\section{Table 1}

Catalpol and its metabolites identified in different human intestinal bacterial samples using UPLC-MS/MS

\begin{tabular}{|c|c|c|c|c|c|c|}
\hline Peak & $t_{\mathrm{R}}(\mathbf{m i n})$ & {$[\mathbf{M}+\mathbf{H}]^{+}(\mathbf{m} / \mathbf{z})$} & $\mathrm{MS}^{2}$ fragment ions $(\mathrm{m} / \mathrm{z})$ & Identification & Formula & Error $(\mathbf{p p m})$ \\
\hline M0 & 0.91 & 363.1292 & $363,201,183,165,147,137,109$ & Catalpol & $\mathrm{C}_{15} \mathrm{H}_{22} \mathrm{O}_{10}$ & -2.0 \\
\hline M1 & 2.95 & 201.3302 & $201,183,165,147,137,109$ & Catalpol aglycone & $\mathrm{C}_{9} \mathrm{H}_{12} \mathrm{O}_{5}$ & 1.3 \\
\hline M2 & 6.41 & 243.4210 & $243,201,183,165,147,137,109$ & Acetylated catalpol & $\mathrm{C}_{11} \mathrm{H}_{14} \mathrm{O}_{6}$ & 0.9 \\
\hline M3 & 4.35 & 247.4360 & $247,229,199,169$ & Dimethylated and hydroxylated catalpol aglycone & $\mathrm{C}_{11} \mathrm{H}_{18} \mathrm{O}_{6}$ & -1.4 \\
\hline M4 & 3.42 & 200.2514 & $200,182,164$ & Nitrogen-containing catalpol aglycone & $\mathrm{C}_{9} \mathrm{H}_{13} \mathrm{O}_{4} \mathrm{~N}$ & 1.0 \\
\hline
\end{tabular}

$t_{\mathrm{R}}$, retention time; $[\mathrm{M}+\mathrm{H}]^{+}$, positive charged molecular ion. 